\title{
A Stochastic Galerkin Cell-based Smoothed Finite Element Method (SGCS-FEM)
}

Tittu Varghese Mathew

Department of Mechanical Engineering, Indian Institute of Technology - Madras, India.

Lars Beex

Faculté des Sciences, de la Technologie et de la Communication, University of Luxembourg, Luxembourg.

lars.beex@uni.lu

Stéphane PA Bordas

Faculté des Sciences, de la Technologie et de la Communication, University of Luxembourg, Luxembourg.

stephane.bordas@uni.lu

Sundararajan Natarajan

Department of Mechanical Engineering, Indian Institute of Technology - Madras, India. snatarajan@iitm.ac.in

Received (Day Month Year)

Revised (Day Month Year)

In this paper, the cell based smoothed finite element method is extended to solve stochastic partial differential equations with uncertain input parameters. The spatial field of Young's moduli and the corresponding stochastic results are represented by KarhunenLoéve expansion and polynomial chaos expansion, respectively. The Young's Modulus of structure is considered to be random for stochastic static as well as free vibration problems. Mathematical expressions and the solution procedure are articulated in detail to evaluate the statistical characteristics of responses in terms of static displacements and free vibration frequencies. The feasibility and effectiveness of the proposed SGCS-FEM method in terms of accuracy and lower requirement on the mesh size in the solution domain over that of conventional FEM for stochastic problems are demonstrated by carefully chosen numerical examples. From the numerical study, it is inferred that the proposed framework is computationally less demanding without compromising accuracy.

Keywords: Stochastic Galerkin Cell-based Smoothed Finite Element Method (SGCSFEM); Karhunen-Loève expansion (KLE); Polynomial Chaos Expansion (PCE); Random Material Properties; Free Vibrations.

\section{Introduction}

The Finite Element Method (FEM) has become one of the most popular numerical techniques to solve practical problems in various fields of engineering. Its only been three decades since the general research community started to extend the potential of the FEM to tackle uncertain systems governed by stochastic PDEs with ran- 
dom input data. The reader may refer to the research monographs by Ghanem and Spanos ${ }^{1}$ and Kleiber and $\mathrm{Hien}^{2}$ for more information. In the stochastic FEM, the uncertain input parameters in the form of material, geometry or loading conditions are represented by using random variables. However, apart from treating the randomness in the input parameters as perturbations about their mean value, most of the uncertainty quantification problems in engineering requires the modeling of spatial variability of the random input parameters. Some examples include: (a) the tensile and the fracture toughness distribution of engineering materials, (b) the uncertain size and shape characteristics of components (small micro-scale inclusions in composites to large components in precision equipment's) and (c) the wind and snow loading on civil and offshore structures.

All the above examples, not only exhibit randomness from sample to sample, but also from point to point in their spatial domain. To enable a computational treatment of such stochastic problems, the random fields are first discretized in order to represent them in a finite number of random variables. In general, the number of random variables must be sufficiently large enough to ensure an accurate representation of the randomness, but the number must be sufficiently small to guarantee manageble computational costs.

Given a system of random algebraic equations, the Monte Carlo simulation techniques can be readily applied to compute the response statistics to a certain degree of accuracy ${ }^{3}$. As per the stochastic mechanics jargon, Monte Carlo techniques are only considered as a last resort, since they are computationally demanding. The perturbation method and Neumann series expansion offer efficient alternative methods to compute the first two statistical moments of the response quantities ${ }^{2,4,5}$. However, a drawback of these local approximation techniques is their inaccuracy if the variation of the random input variables increases ${ }^{6}$. In the work of Ghanem and Spanos $^{7}$, a spectral stochastic FEM is proposed, in which the random algebraic equations are solved using the Wiener Polynomial Chaos (PC) decomposition method. The basic principle behind this approach is to represent the stochastic output in terms of a linear combination of multi-dimensional Hermite polynomials of which the coefficients are to be solved for. These coefficients can be uniquely determined by employing the Galerkin projection scheme. Based on the advantages of such projection schemes, a wide variety of stochastic mechanics problems have been solved in the last decade, for example elasticity problems ${ }^{1}$, random vibration problems ${ }^{8}$, soil mechanics with random interfaces ${ }^{9}$, elasto-plastic material problems ${ }^{10}$, to name a few.

A number of methods exists in discretizing the random fields. Interested readers may refer to the work of Betz ${ }^{11}$ for a summary of existing techniques for the same and the references therein. A popular approach that is used in the current study is the Karhunen-Loève expansion, which involves the spectral decomposition of the covariance functions. This process produces a representation of random field in the form of an infinite series of deterministic spatial functions and uncorrelated random variables. 
Up until now, a brief overview of the random field discretization and the projection of stochastic output response over polynomial basis have been reviewed. While basis functions from conventional FEM are used to discretise the eigenvectors of a given covariance function, several research efforts have gone in parallel to improve the accuracy of the statistical moments of the stochastic outputs using various advanced numerical techniques. For example, Long ${ }^{12}$ extended the scaled boundary finite element formulation to random parameter field problems in which the perturbation method was used to determine the statistical moments. Arun ${ }^{13}$ used mesh free methods in to solve boundary-value problems in damage mechanics under elasto-plastic conditions. Keyan $\mathrm{Li}^{14}$ used non-uniform rational B-spline (NURBS) and T-spline basis functions for meshing both the stochastic and the spatial domain for complex geometries. The shared denominator between the FEM and the mesh-free methods is that they are rooted under the same Galerkin formulation, but their function spaces and basis functions are different. As the problem complexity increases, lower order elements are commonly preferred, but comes with the limitation of an reduced inefficiency for many practical applications. For example, lower order elements exhibit an overestimation of the stiffness matrix and as a byproduct, an underestimation of the internal strain energy. Furthermore, the element shape cannot be distorted beyond a certain limit due to the limitation of the mapping between the parent and the physical domain.

To overcome aforementioned issues, inspired by the work of Yoo et al. ${ }^{15}$, Liu et al., ${ }^{16}$ proposed the Smoothed Finite Element Method (SFEM). For recent developments of the SFEM and its applications, readers are referred to the recent review paper ${ }^{17}$. Liuet al., ${ }^{16}$ extended the cell based SFEM for stochastic analysis based on generalized stochastic perturbation technique. Huet al., ${ }^{18}$ generalized the $n^{\text {th }}$ order stochastic perturbation technique based on a stable node-based smoothed finite element method. Wuet al., ${ }^{19}$ applied the generalized edge based smoothed finite element for stochastic analysis of Reissner Mindlin plates, with the aim of overcoming the shortcomings of conventional $2^{\text {nd }}$ order perturbation approach with small perturbations. Wuet al., ${ }^{20}$ introduced the first order perturbation technique into the edge-based smoothed FEM for probabilistic analysis of structural acoustic systems. To the best of knowledge of the authors, there exists no literature that deals with random field representation within a smoothed finite element setting.

In this paper, a novel stochastic formulation using smoothed FEM, namely Stochastic Galerkin Cell-based Smoothed Finite Element Method (SGCS-FEM) is presented in detail. Numerical studies are presented for two-dimensional elasticity problems (stochastic static displacements and an eigenvalue problems) and a threedimensional problem with parametric uncertainty. The results are presented for the first two statistical moments of displacements in all the examples obtained using the novel method and are compared with MCS results.

The remainder of this paper is organized as follows. Section 2 presents the governing equations, the corresponding weak form and a description to represent the random input variable. An overview of the cell-based SFEM is given in Section 
3. The novel stochastic Galerkin SFEM and the solution schemes for static and free vibration problems are discussed in Section 4. Section 5 demonstrates the accuracy of the proposed framework with a few benchmark problems. The relative accuracy obtained using the proposed method and that from using the conventional FEM in terms of error in the mean and the standard deviation predictions are discussed in detail, followed by concluding remarks in the last section.

\section{Governing equations and weak form}

Consider a homogeneous linear elastic body occupying domain $\mathcal{S} \subset \mathbb{R}^{d}$, where $d=$ 2,3 . The boundary $(\partial \mathcal{S})$ is considered to admit the decomposition with the outward normal $\mathbf{n}$ into disjoint sets, i.e., $\partial \mathcal{S}=\partial \mathcal{S}_{\mathrm{D}} \cup \partial \mathcal{S}_{\mathrm{t}}$ and $\partial \mathcal{S}_{\mathrm{D}} \cap \partial \mathcal{S}_{\mathrm{t}}=\emptyset$, where on $\partial \mathcal{S}_{\mathrm{D}}$ and $\partial \mathcal{S}_{\mathrm{t}}$, Dirichlet boundary and Neumann boundary conditions are specified. In the absence of body forces, the coupled governing equations for linear elasto-statics undergoing small deformation is: find $(\mathbf{u}): \mathcal{S} \rightarrow \mathbb{R}^{d}$ such that

$$
\boldsymbol{\nabla} \cdot \boldsymbol{\sigma}=\rho \ddot{\mathbf{u}} \text { in } \mathcal{S},
$$

with the following boundary conditions:

$$
\begin{array}{rlll}
\boldsymbol{\sigma} \cdot \mathbf{n} & =\overline{\mathbf{t}} & \text { on } & \partial \mathcal{S}_{\mathrm{t}}, \\
\mathbf{u} & =\overline{\mathbf{u}} & \text { on } & \partial \mathcal{S}_{\mathrm{D}},
\end{array}
$$

where $\rho$ is the mass density, $\overline{\mathbf{t}}$ and $\overline{\mathbf{u}}$ are the externally applied traction and displacements, respectively, $\mathbf{n}$ is the unit outward normal and $\boldsymbol{\sigma}$ is the Cauchy stress related to the small strain $\varepsilon$ by

$$
\sigma=\mathrm{D} \varepsilon
$$

where $\mathbf{D}$ is the material constitutive matrix and $\varepsilon=\frac{1}{2}\left(\nabla \mathbf{u}^{\mathrm{T}}+\nabla \mathbf{u}\right)$. By following the standard Galerkin procedure, the corresponding weak form is given by: find $\mathbf{u} \in \mathscr{U}$ such that:

$$
\int_{\mathcal{S}} \boldsymbol{\sigma}(\mathbf{u}): \varepsilon(\mathbf{v}) \mathrm{d} \mathcal{S}+\int_{\partial \mathcal{S}_{t}} \overline{\mathbf{t}} \cdot \mathbf{v} \mathrm{d} \partial \mathcal{S}+\int_{\Omega} \mathbf{v} \rho \ddot{\mathbf{u}} \mathrm{d} \mathcal{S}=\mathbf{0}
$$

where

$$
\begin{aligned}
& \mathscr{U}(\mathcal{S})=\left\{\mathbf{u}: \mathcal{S} \rightarrow \mathbb{R}^{d} \mid u_{I} \in H^{1}(\mathcal{S}), I=1, \cdots, d, \mathbf{u}=\overline{\mathbf{u}} \text { on } \partial \mathcal{S}_{u}\right\} \\
& \mathscr{V}(\mathcal{S})=\left\{\mathbf{v}: \mathcal{S} \rightarrow \mathbb{R}^{d} \mid v_{I} \in H^{1}(\mathcal{S}), I=1, \cdots, d, \mathbf{v}=\mathbf{0} \text { on } \partial \mathcal{S}\right\}
\end{aligned}
$$

are the displacement trial and the test function space, respectively, and $H^{1}$ denotes the Hilbert-Sobolev first order space.

In this study, the Young's Modulus is modeled as a random field, say $\mathrm{E}(\mathbf{x} ; \omega)$. In other words, for each $\mathbf{x} \in \mathbb{R}^{2}, \mathrm{E}: \Omega \rightarrow \mathbb{R}$ is a random variable on a probability space $(\Omega, \Im, \Gamma)$, where $\Omega$ is the set of elementary events, $\Im$ is the $\sigma$-algebra associated with $\Omega$ and $\Gamma$ is a probability measure. We use $\omega$ to indicate the dependence of a quantity on the random dimension of the problem. Since the Young's Modulus is 
treated to be a random field, the elasticity matrix becomes a function of the spatial coordinate and a random dimension $(\omega)$, and can be represented as follows:

$$
\mathbf{D}(\mathbf{x} ; \omega)=\mathrm{E}(\mathbf{x} ; \omega) \mathbf{D}_{0}
$$

where $\mathbf{D}_{0}$ is the deterministic part of the elasticity matrix. Random field discretization is a key step in the numerical solution of stochastic PDEs. Various discretization techniques are available in the literature to approximate the random fields including the mid-point method, shape function methods, optimal linear estimation, weighted integral methods, orthogonal series expansion and Karhunen-Loève (KL) expansion scheme. For a detailed overview and comparison of these methods, the reader is referred to ${ }^{21,22}$. In our present study, the KL expansion technique is used to discretize random fields.

Let the covariance function of a random field $\mathrm{E}(\mathbf{x} ; \omega)$ be $\mathbf{R}_{\mathrm{E}}\left(\mathbf{x}_{1}, \mathbf{x}_{2}\right)$. A typical example for an exponential covariance function between two points $\mathbf{x}_{1}=\left(x_{1}, y_{1}, z_{1}\right)$ and $\mathbf{x}_{2}=\left(x_{2}, y_{2}, z_{2}\right)$, commonly found and quoted in literature is shown below:

$$
\mathbf{R}_{\mathrm{E}}\left(\mathbf{x}_{1}, \mathbf{x}_{2}\right)=\exp \left(-\left|\frac{x_{1}-x_{2}}{\ell_{c x}}\right|-\left|\frac{y_{1}-y_{2}}{\ell_{c y}}\right|-\left|\frac{z_{1}-z_{2}}{\ell_{c z}}\right|\right)
$$

Then, the KL expansion of the random field $\mathrm{E}(\mathbf{x} ; \omega)$ can be written as

$$
\mathrm{E}(\mathbf{x} ; \omega)=\langle\mathrm{E}(\mathbf{x} ; \omega)\rangle+\sum_{i=1}^{\infty} \sqrt{\lambda_{i}} \sigma \theta_{i} \mathrm{E}_{i}(\mathbf{x})
$$

where $\lambda_{i}$ and $h_{i}(x)$ are the eigenvalues and eigenfunctions of $\mathbf{R}_{\mathrm{E}}\left(\mathbf{x}_{1}, \mathbf{x}_{2}\right)$, respectively, $\theta_{i}, i=1,2, \ldots, \infty$ are a set of uncorrelated random variables, $\sigma$ denotes the standard deviation and $\langle\cdot\rangle$ denotes the expectation operator. The eigenvalues and eigenfunctions of the covariance function can be computed using a Fredholm integral equation of the second kind, i.e.,

$$
\int_{\Omega} \mathbf{R}_{\mathrm{E}}\left(\mathbf{x}_{1}, \mathbf{x}_{2}\right) \mathrm{E}_{i}\left(\mathbf{x}_{1}\right) d \mathbf{x}_{1}=\lambda_{i} \mathrm{E}_{i}\left(\mathbf{x}_{2}\right)
$$

Analytical solutions of the above integral eigenvalue problem can be obtained only for a special class of functions (e.g., the exponential covariance function) defined on geometrically simple domain. However, for more general cases, numerical discretization schemes have to be employed to compute the eigenvalues and eigenfunctions of $\mathbf{R}_{\mathrm{E}}\left(\mathbf{x}_{1}, \mathbf{x}_{2}\right)$. This procedure is discussed in Appendix 1. Truncating Equation (2.7) at the $M$ th term gives a finite-dimensional approximation of the random field as

$$
\hat{\mathrm{E}}(\mathbf{x} ; \omega)=\langle\mathrm{E}(\mathbf{x} ; \omega)\rangle+\sum_{i=1}^{M} \sqrt{\lambda_{i}} \sigma \theta_{i} \mathrm{E}_{i}(\mathbf{x})
$$

Substitution of the discretized random field representation into Equation (2.5) results in a representation of the elasticity matrix in terms of a finite number of random variables. 


\section{Overview of the CS-FEM}

The basic idea within the framework of the SFEM is to write a 'smoothed' strain field as a spatial average of the compatible strain field. Mathematically,

$$
\tilde{\varepsilon}^{h}\left(\mathbf{x}_{c}\right)=\int_{\Omega_{h}} \varepsilon_{i j}^{h}(\mathbf{x}) \varphi\left(\mathbf{x}-\mathbf{x}_{c}\right) d \mathbf{x}
$$

where $\varphi$ is a smoothing function that generally satisfies the following properties ${ }^{23}$

$$
\varphi(\mathbf{x}) \geq 0 \quad \forall \mathbf{x} \quad \text { and } \quad \int_{\Omega^{h}} \varphi(\mathbf{x}) \mathrm{d} \Omega=1
$$

Of the different variants of the SFEM, in this study we adopt the cell-based SFEM. Within this framework, the finite element is sub-divided into 'smoothing cells' and the smoothed strain field is computed over the smoothing cells. Figure 1 a schematic representation of the smoothing cells for two and three dimensional elements. It is noted that the purpose of sub-division is to compute the smoothed strain field and it does not add any additional degrees of the freedom. For sake of brevity, the smoothed strain matrix computation is detailed only for two dimensions. Its extension to three dimensions is considered to be straight forward here and interested readers are referred to ${ }^{24,25}$, for detailed derivation and implementation aspects. To use Equation (3.1), the subcell containing point $\mathbf{x}_{c}$ must be first located in order to compute the correct value of the weight function $\varphi$. The discretized strain field is computed through the so-called smoothed discretised gradient operator $\tilde{\mathbf{B}}$, defined by:

$$
\tilde{\epsilon}^{h}\left(\mathbf{x}_{c}\right)=\tilde{\mathbf{B}}_{c}\left(\mathbf{x}_{c}\right) \mathbf{u}
$$

where $\mathbf{u}$ contains the unknown nodal displacements of a finite element. The smoothed element stiffness matrix for an element $e$ is computed by adding up the contributions from each of the subcells within that element

$$
\tilde{\mathbf{K}}^{e}=\sum_{C=1}^{n c} \int_{\Omega_{C}} \tilde{\mathbf{B}_{C}}{ }^{T} \mathbf{D} \tilde{\mathbf{B}_{C}} d \Omega=\sum_{C=1}^{n c}{\tilde{\mathbf{B}_{C}}}^{T} \mathbf{D} \tilde{\mathbf{B}_{C}} \int_{\Omega_{c}} d \Omega=\sum_{C=1}^{n c} \tilde{\mathbf{B}}_{C}^{T}{ }^{T} \tilde{\mathbf{B}} A_{C}
$$

where $n c$ is the number of smoothing subcells in the element. The strain displacement matrix $\tilde{\mathbf{B}}_{C}$ is treated to be constant over each subcell $\Omega_{C}$ and is of the following form

$$
\tilde{\mathbf{B}}_{C}=\left[\begin{array}{lllll}
\tilde{\mathbf{B}}_{C 1} & \tilde{\mathbf{B}}_{C 2} & \tilde{\mathbf{B}}_{C 3} & \cdots & \tilde{\mathbf{B}}_{C n}
\end{array}\right]
$$

where for all the shape functions $I \in\{1,2, \ldots, n\}$, the $3 \times 2$ submatrix $\tilde{\mathbf{B}}_{C I}$ represents the contribution to the strain displacement matrix associated with shape function $I$ and cell $\mathrm{C}$ and writes

$$
\begin{aligned}
\forall I \in\{1,2, \ldots, n\}, \forall C \in\{1,2, \ldots, n c\} \quad \tilde{\mathbf{B}}_{C I} & =\frac{1}{A_{C}} \int_{S_{C}} \mathbf{n}^{T}(\mathbf{x}) N_{I}(\mathbf{x}) d S \\
& =\frac{1}{A_{C}} \int_{S_{C}}\left[\begin{array}{cc}
n_{x} & 0 \\
0 & n_{y} \\
n_{y} & n_{x}
\end{array}\right](\mathbf{x}) N_{I}(\mathbf{x}) d S
\end{aligned}
$$




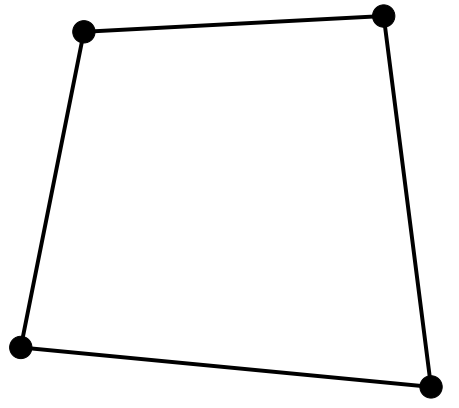

(a)

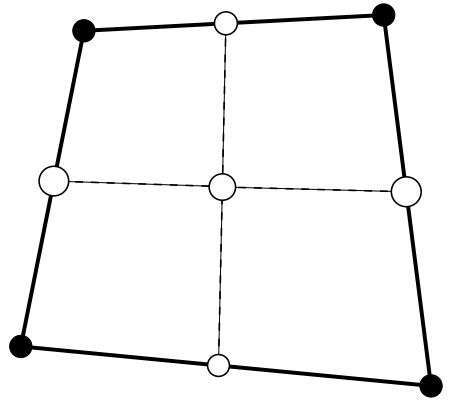

(b)

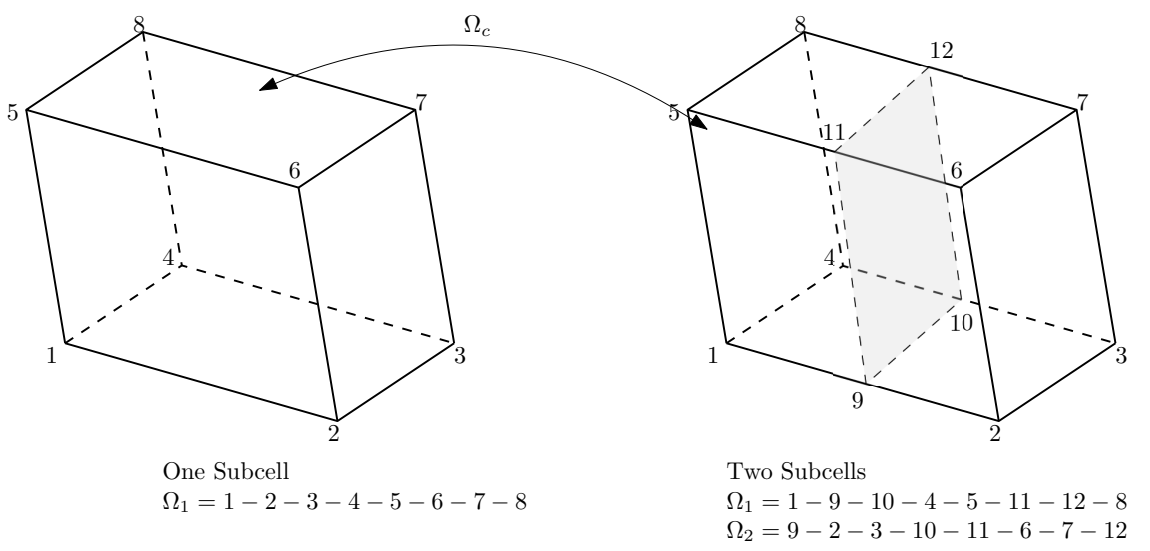

(c)

Fig. 1. Subdivision of an eight noded trilinear hexahedral element and four noded quadrilateral elements into subcells. Note that the subdivision is solely for the purpose of numerical integration and does not introduce additional degrees of freedom.

Only one Gauss point is sufficient for an exact integration of the above equation along the boundary of the subcell $\Omega_{C}$.

$$
\tilde{\mathbf{B}}_{C I}\left(\mathbf{x}_{C}\right)=\frac{1}{A_{C}} \sum_{b=1}^{n b}\left[\begin{array}{cc}
N_{I}\left(\mathbf{x}_{b}^{C}\right) n_{x} & 0 \\
0 & N_{I}\left(\mathbf{x}_{b}^{C}\right) n_{y} \\
N_{I}\left(\mathbf{x}_{b}^{C}\right) n_{y} & N_{I}\left(\mathbf{x}_{b}^{C}\right) n_{x}
\end{array}\right] l_{b}^{C}
$$

where $\mathbf{x}_{b}^{C}$ and $l_{b}^{C}$ are the center point coordinates and the length of a boundary of subcell, $\Gamma_{b}^{C}$ respectively. The procedure outlined so far is general and is applicable to polygons of arbitrary shapes. However, in the forthcoming studies, we will be limiting ourselves to quadrilateral elements, so as to facilitate the comparison of the performance of the stochastic CSFEM with the conventional FEM. As a result of strain smoothing, only the computations of shape functions are involved in the evaluation of the field gradients and thereby the stiffness matrix. The shape functions at the Gauss points along each edge of a sub-cell are computed as the average of the shape function values at the two ends of the edge. 


\section{Stochastic Galerkin Smoothed Finite Element formulation}

In this section, we derive the stochastic stiffness matrix, the mass matrix and briefly discuss the solution procedure adopated to compute the stochastic response. Unless mentioned otherwise, the random field is assumed to be homogeneous with constant mean across the domain. Upon substituting Equation (2.9) into Equation (3.4), the smoothed element stiffness matrix with a random Young's Modulus can be written as:

$$
\begin{aligned}
\tilde{\mathbf{K}^{e}=} & \sum_{C=1}^{n c} \tilde{\mathbf{B}_{C}}{ }^{T} \mathbf{D}(\mathbf{x} ; \omega) \tilde{\mathbf{B}_{C}} A_{C} \\
= & \sum_{C=1}^{n c} \tilde{\mathbf{B}_{C}}{ }^{T}\left(\sum_{i=0}^{M} \mathrm{E}_{i} \theta_{i}\right) \tilde{\mathbf{D}_{0}} \tilde{\mathbf{B}_{C}} A_{C} \\
= & \sum_{i=0}^{M} \sum_{C=1}^{n c} \mathrm{E}_{i} \tilde{\mathbf{B}_{C}}{ }^{T} \tilde{\mathbf{D}}_{0} \tilde{\mathbf{B}_{C}} A_{C} \theta_{i} \\
= & \sum_{C=1}^{n c}\left\langle\mathrm{E}\left(x_{0}^{C}, y_{0}^{C} ; \omega\right){\tilde{\mathbf{B}_{C}}}^{T} \mathbf{D}_{0} \tilde{\mathbf{B}_{C}} A_{C}\right. \\
& +\sum_{i=1}^{M} \sum_{C=1}^{n c} \tilde{\mathbf{B}_{C}}{ }^{T} \sqrt{\lambda_{i}} \theta_{i} \sigma \mathrm{E}_{i}\left(x_{0}^{C}, y_{0}^{C}\right) \mathbf{D}_{0} \tilde{\mathbf{B}_{C}} A_{C} \\
= & \tilde{\mathbf{K}}_{0}^{e}+\sum_{i=1}^{M} \tilde{\mathbf{K}_{i}^{e}} \theta_{i}
\end{aligned}
$$

During the assembly process over the subcells while defining an elemental stiffness

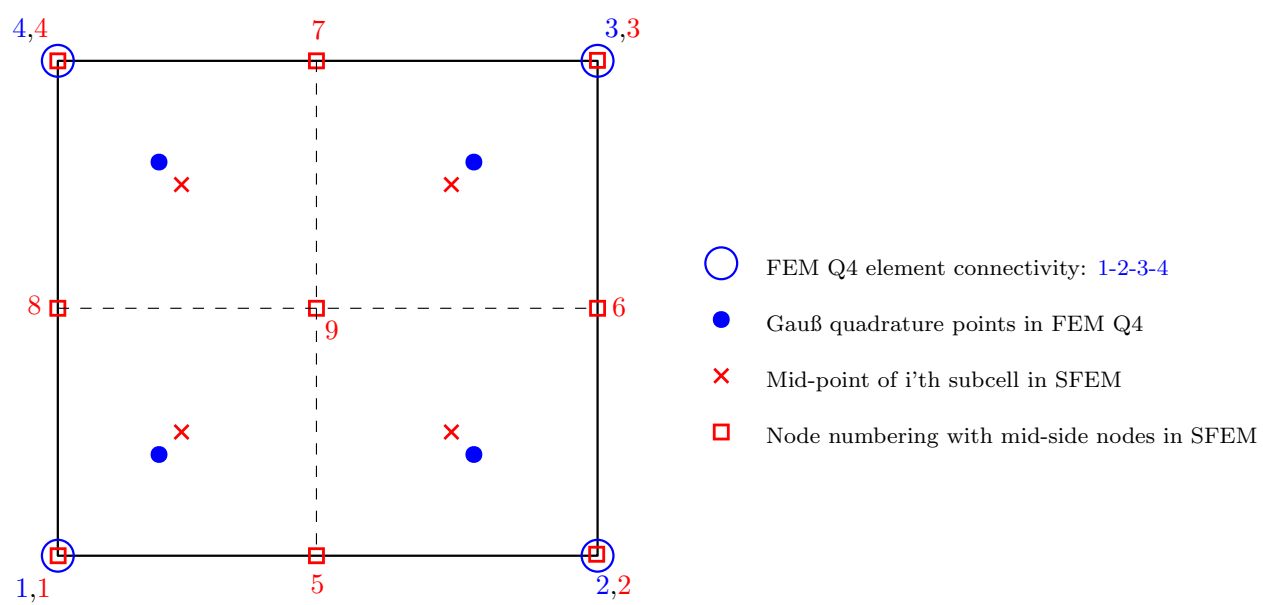

Fig. 2. Nodal connectivity of a conventional FEM Q4 element and SFEM with 4 subcells. Internal points marked as $\times$ are cell mid-points where the random constitutive matrix $\mathbf{D}\left({ }^{`}\right)$ are evaluated in the case of SGCS-FEM. Whereas, the $\bullet$ represents the Gauss quadrature points in an FEM Q4 element (full integration) where the same random constitutive matrix $\mathbf{D}(\theta)$ is evaluated 


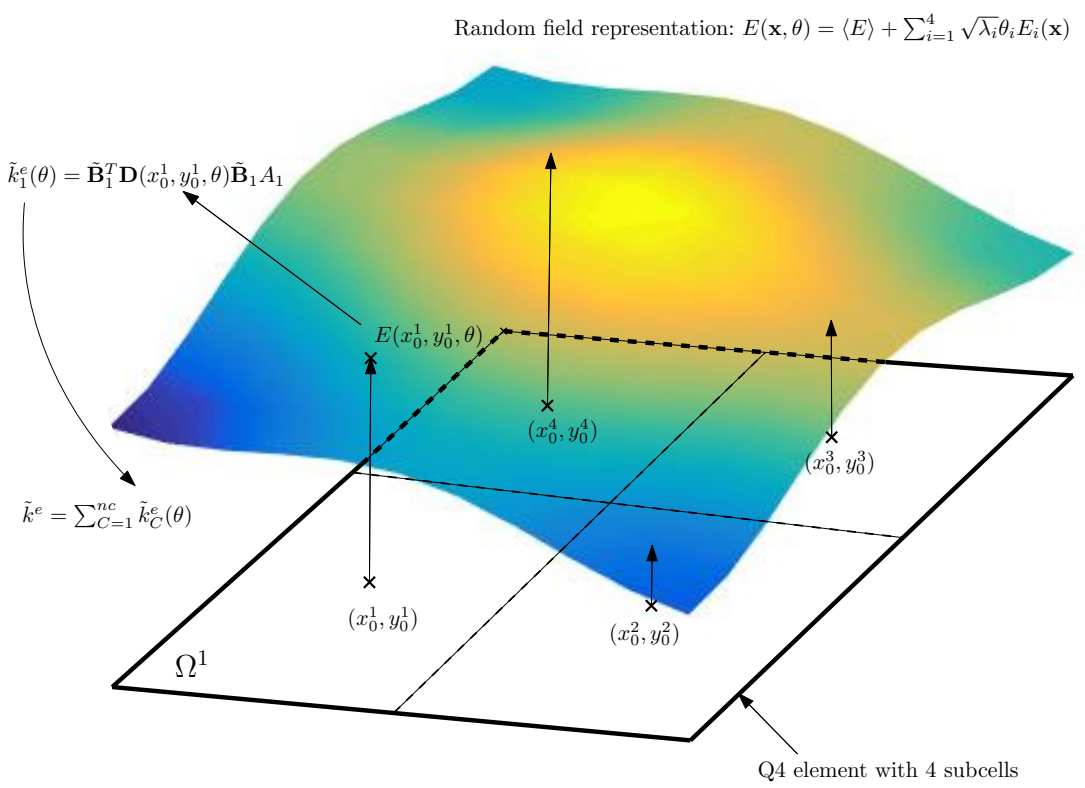

Fig. 3. Projection scheme between the solution and stochastic domain for a smoothed element with 4 subcells.

matrix, the centroid of the $C^{r m t h}$ sub-cell, namely $\left(x_{0}^{C}, y_{0}^{C}\right)$ is projected onto the random field, as shown schematically in Figure 3. In juxtaposing this projection scheme with that from a stochastic FEM with random material field domains, we see that the projection points, namely the Gauss quadrature points in the FEM with full integration and the centroids of the subcell are not far apart, as illustrated schematically in Figure 2. The mass matrix is given by:

$$
\mathbf{M}^{e}=\int_{\Omega^{e}} \mathbf{N}^{\mathrm{T}} \rho \mathbf{N} \mathrm{d} \Omega
$$

where $\mathbf{N}$ is the matrix of shape functions.

\subsection{Static case}

For static case, the assembly of the smoothed element stiffness matrices results in the following system of linear random algebraic equations:

$$
\left(\tilde{\mathbf{K}}_{0}+\sum_{i=1}^{M} \tilde{\mathbf{K}}_{i} \theta_{i}\right) \mathbf{u}(\theta)=\mathbf{f} \text { or }\left(\sum_{i=0}^{M} \tilde{\mathbf{K}}_{i} \theta_{i}\right) \mathbf{u}(\theta)=\mathbf{f}
$$


where $\tilde{\mathbf{K}}_{0} \in \mathbb{R}^{n \times n}$ and $\tilde{\mathbf{K}}_{i} \in \mathbb{R}^{n \times n}$ are deterministic stiffness matrices, $\mathbf{u}(\theta) \in \mathbb{R}^{n}$ is the random displacement vector, $\theta_{0}=1, \mathbf{f} \in \mathbb{R}^{n}$ denotes the force vector which is assumed to be deterministic for simplicity reasons and $n$ is the total number of degrees of freedom. The vector of unknown random displacement vector is computed by employing the polynomial chaos projection scheme ${ }^{1}$.

In this scheme, the random nodal displacements are expanded using a set of multidimensional Hermite polynomials for Gaussian input data, which results in the following expansion for the random process

$$
\mathbf{u}(\theta)=\sum_{i=0}^{P-1} \mathbf{u}_{i} \Psi_{i}(\theta)
$$

where $\mathbf{u}_{i} \in \mathbb{R}^{n}, i=0,1,2, \ldots, P-1$, are sets of vectors formed from the undetermined coefficients in the PC expansion for each nodal displacement. $\Psi_{j}(\theta)$ is a set of orthogonal Hermite polynomials. The number of terms in the expansion, $\mathrm{P}$, is given by

$$
P=\frac{(M+p) !}{p !(M) !}
$$

where $p$ is the highest order of the set of Hermite polynomials $\Psi_{j}$. Substitution of the $\mathrm{PC}$ expansion of $\mathbf{u}(\theta)$ into the governing random algebraic equations given in Equation (4.3) gives

$$
\left(\sum_{i=0}^{M} \tilde{\mathbf{K}}_{i} \theta_{i}\right)\left(\sum_{j=0}^{P-1} \mathbf{u}_{j} \Psi_{j}(\theta)\right)=\mathbf{f}
$$

The undetermined terms in the PC expansion can be uniquely computed using the Galerkin projection condition, which involves orthogonalizing the stochastic residual error to the approximating random subspace as shown below ${ }^{1}$

$$
\left\langle\epsilon(\theta), \Psi_{k}(\theta)\right\rangle=0, \quad k=0,1,2, \ldots, P-1
$$

where the stochastic residual $\epsilon(\theta)$ is given by

$$
\epsilon(\theta)=\left(\sum_{i=0}^{M} \tilde{\mathbf{K}}_{i} \theta_{i}\right)\left(\sum_{j=0}^{P-1} \mathbf{u}_{j} \Psi_{j}(\theta)\right)-\mathbf{f}
$$

Substituting Equation (4.8) into Equation (4.7) results in the following systems of deterministic equations:

$$
\sum_{i=0}^{M} \sum_{j=0}^{P-1} \tilde{\mathbf{K}}_{i} \mathbf{u}_{j}\left\langle\theta_{i} \Psi_{j} \Psi_{k}\right\rangle=\left\langle\Psi_{k} \mathbf{f}\right\rangle, \quad k=0,1,2, \ldots, P-1
$$

The expectation operations in Equation (4.9) can be readily carried out using the properties of Wiener Hermite chaos ${ }^{1,21}$. Now, expanding the above equation 
about the subscripts $j$ and $k$, we arrive at the following system of linear algebraic equations:

$$
\left[\begin{array}{cccc}
\tilde{\mathbf{K}}_{0,0} & \tilde{\mathbf{K}}_{0,1} & \cdots & \tilde{\mathbf{K}}_{0, P-1} \\
\tilde{\mathbf{K}}_{1,0} & \tilde{\mathbf{K}}_{1,1} & \cdots & \tilde{\mathbf{K}}_{1, P-1} \\
\vdots & \vdots & \ddots & \vdots \\
\tilde{\mathbf{K}}_{P-1,0} & \tilde{\mathbf{K}}_{P-1,1} & \cdots & \tilde{\mathbf{K}}_{P-1, P-1}
\end{array}\right]\left[\begin{array}{c}
\mathbf{u}_{0} \\
\mathbf{u}_{1} \\
\vdots \\
\mathbf{u}_{P-1}
\end{array}\right]=\left[\begin{array}{c}
\mathbf{f}_{0} \\
\mathbf{f}_{1} \\
\vdots \\
\mathbf{f}_{P-1}
\end{array}\right]
$$

which is of the form $\tilde{\mathbf{K}} \hat{\mathbf{u}}=\hat{\mathbf{f}}$, where $\tilde{\mathbf{K}} \in \mathbb{R}^{n P \times n P}$ and $\hat{\mathbf{u}}, \hat{\mathbf{f}} \in \mathbb{R}^{n P}$.

Remark 4.1 It can be noted that the computational complexity and memory requirements of PC projection scheme increases significantly when $M$ and $p$ are increased. However, the memory requirements can be reduced by precomputing and storing the expectation terms of the form $\left\langle\theta_{i} \Psi_{j} \Psi_{k}\right\rangle$. Furthermore, the sparsity of the tensor products can also be exploited to accelerate the stochastic computations. In the interest of the reader, a detailed overview of numerical schemes which exploits the sparse structure of equation Equation (4.10) can be found in the literature ${ }^{26,27}$.

After solving Equation (4.10) and substituting back the results in Equation (4.4), one arrives at an explicit expression for the response process. This enables the statistics of the displacements as well as other response quantities of interest to be computed efficiently in the post-processing phase. For example, the mean (first moment) of displacements is computed as

$$
\langle\mathbf{u}(\mathbf{x}, \omega)\rangle=\mathbf{u}_{0}(\mathbf{x})
$$

and the standard deviation (square root of the second moment about the mean) is

$$
\operatorname{stdev}(\mathbf{u}(\mathbf{x}, \omega))=\sqrt{\left\langle\left(\sum_{i=1}^{P-1} \mathbf{u}_{i}(\mathbf{x}) \Psi_{i}(\theta)\right)^{2}\right\rangle}
$$

which, when using the orthogonality property of Wiener-Hermite chaos, simplifies to

$$
\operatorname{stdev}(\mathbf{u}(\mathbf{x}, \omega))=\sqrt{\left(\sum_{i=1}^{P-1} \mathbf{u}_{i}^{2}(\mathbf{x})\left\langle\Psi_{i}^{2}(\theta)\right\rangle\right)^{2}}
$$

\subsection{Free vibration}

In this case, the assembly of the smoothed element stiffness matrices and the mass matrix results in the following system of linear random algebraic equations:

$$
\tilde{\mathbf{K}}(\theta) \mathbf{u}(\theta)+\mathbf{M} \ddot{\mathbf{u}}(\theta)=\mathbf{0}
$$

Upon substituting $\mathbf{u}(\theta)=\boldsymbol{\delta}(\theta) e^{-i \lambda(\theta) t}$, we get:

$$
[\tilde{\mathbf{K}}(\theta)-\lambda(\theta) \mathbf{M}] \boldsymbol{\delta}(\theta)=\mathbf{0}
$$


where

$$
\tilde{\mathbf{K}}(\theta)=\sum_{i=0}^{M} \tilde{\mathbf{K}}_{i} \theta_{i}
$$

is the stochastic stiffness matrix. Using Equation (4.16), Equation (4.15) can be rewritten as:

$$
[\tilde{\mathbf{R}}(\theta)-\lambda(\theta)] \boldsymbol{\delta}(\theta)=\mathbf{0}
$$

where, $\tilde{\mathbf{R}}(\theta)=\mathbf{M}^{-1} \tilde{\mathbf{K}}(\theta)=\mathbf{M}^{-1} \sum_{i=0}^{M} \tilde{\mathbf{K}}_{i} \theta_{i}$. Equation (4.17) is a stochastic eigenvalue problem and in this study, the stochastic subspace inverse iteration method is used to solve for the eigenvalues and the eigenvectors. As the covariance of the eigenvalues and the eigenvectors are not known, these are represented by using a truncated PC expansion as:

$$
\begin{aligned}
\lambda(\theta) & =\sum_{j=0}^{P-1} \lambda_{j} \Psi_{j}(\theta) \\
\boldsymbol{\delta}(\mathbf{x}, \theta) & =\sum_{j=0}^{P-1} \boldsymbol{\delta}_{j} \Psi_{j}(\theta)
\end{aligned}
$$

where $\Psi_{j}(\theta)$ are the Hermite polynomials, $P$ is the number of terms in a truncated $\mathrm{PC}$ expansion as given in Equation (4.5). The first step is to solve the deterministic eigenvalue problem and compute the first (lowest) fundamental eigenvalue and eigenvector, which is used as the initial guess for the Stochastic Subspace Inversion Method (SSIM) ${ }^{28}$. In this study, the QR algorithm is employed to solve the deterministic eigenvalue problem. The SSIM computes a correction of the expected value of the eigenvector from the mean and the coefficients of the terms in KL expansion. Similar to the deterministic inverse subspace method, after each iteration, the eigenvectors have to be normalized. The eigenvectors considered here (c.f. Equation (4.18)) have the form of stochastic polynomials. This makes it feasible to sample a given random vector, $\theta$ using a discrete projection rule combined with collocation points ${ }^{28}$. The eigenvalue expansion coefficients, $\lambda_{j}$ (c.f. Equation (4.18)) are computed from the stochastic Rayleigh quotient through a discrete projection, given by:

$$
\begin{aligned}
\lambda_{k} & =\left\langle\lambda, \Psi_{k}\right\rangle \quad k=0, \cdots, P-1 \\
& =\frac{1}{\left\langle\Psi_{k}, \Psi_{k}\right\rangle} \sum_{j=0}^{P-1} \sum_{i=0}^{P-1} \sum_{\ell=0}^{M} c_{i j k \ell} \boldsymbol{\delta}_{i}^{\mathrm{T}} \tilde{\mathbf{R}}_{\ell} \boldsymbol{\delta}_{j} ; \quad c_{i j k \ell}=\left\langle\psi_{i}, \zeta_{\ell}, \psi_{j}, \psi_{k}\right\rangle
\end{aligned}
$$

These steps are detailed in Algorithm 1.

\section{Numerical examples}

In this section, the stochastic response of a few two and three dimensional problems are studied to demonstrate the efficiency of the proposed framework. The first two 


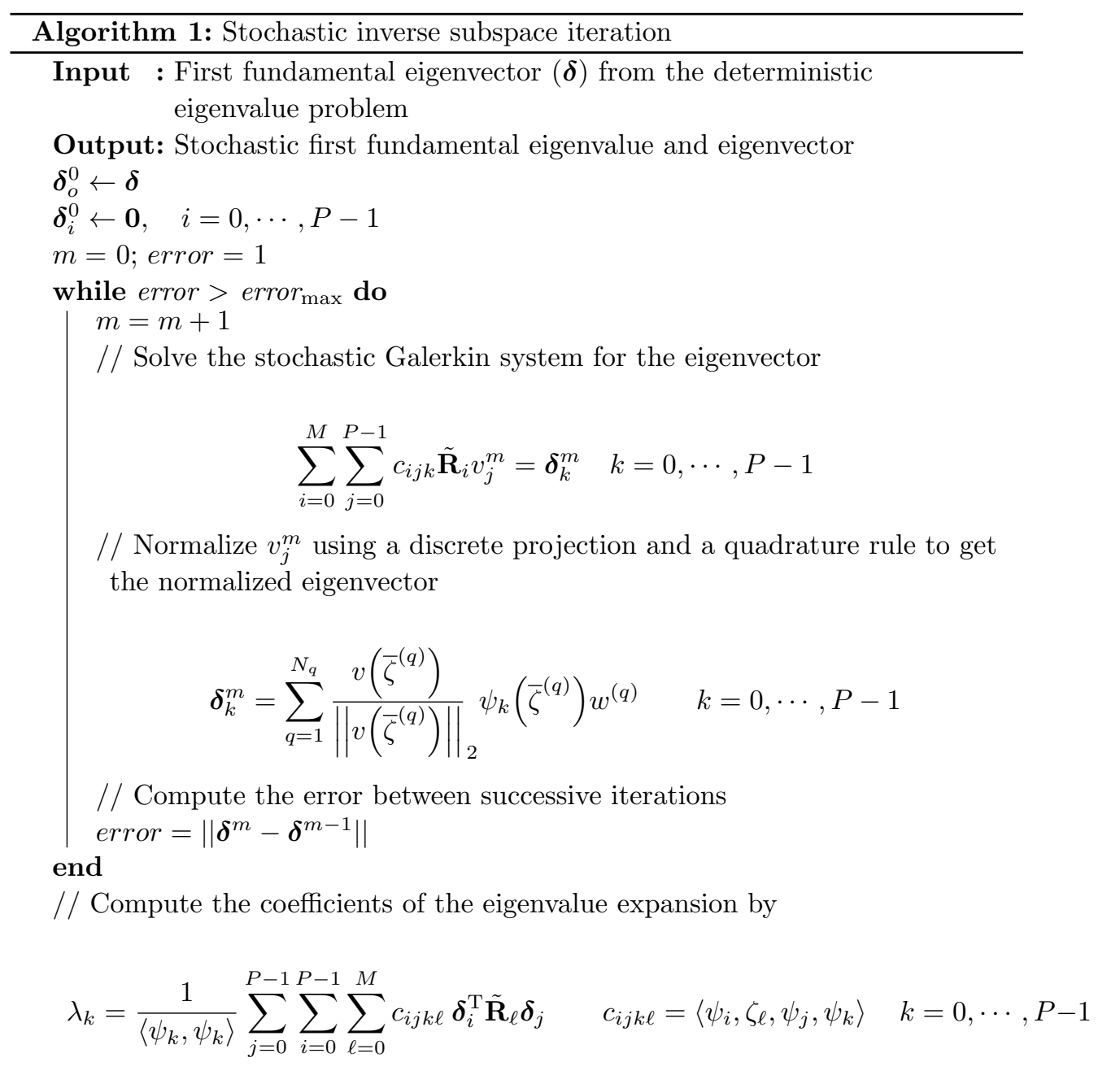

statistical moments of the displacements using the proposed method is compared with thos obtained using deterministic MC simulations and with the conventional FEM. The two and the three dimensional domains are discretized with four noded bilinear quadrilateral and eight noded trilinear hexahedral elements, respectively. Table 1 shows the convention adopted to compute the terms in the stiffness matrix. To study the convergence properties of the SGCS-FEM and the FEM, the following 
Table 1. Number of integration points and subcells employed to compute the terms in the stiffness matrix in case of the FEM and SGCS-FEM, respectively.

\begin{tabular}{llcc}
\hline Dimensions & Element Type & $\begin{array}{c}\text { FEM } \\
\text { (number of integration points) }\end{array}$ & $\begin{array}{c}\text { CS-FEM } \\
\text { (number of subcells) }\end{array}$ \\
\hline Two & Four noded quadrilateral & 4 & 4 \\
Three & Eight noded hexahedral & 8 & 8 \\
\hline
\end{tabular}

error norms are used:

$$
\begin{aligned}
& \text { Error }_{\text {mean }}=\frac{\sum_{i=1}^{n \text { dof }}\left|U_{i}^{\text {mean }}-U_{i}^{M C S}\right|}{\sum_{i=1}^{n d o f}\left|U_{i}^{M C S}\right|} \\
& \text { Error }_{\text {stdev }}=\frac{\sum_{i=1}^{n d o f}\left|U_{i}^{\text {stdev }}-U_{i}^{M C S}\right|}{\sum_{i=1}^{\text {ndof }}\left|U_{i}^{M C S}\right|}
\end{aligned}
$$

where $U_{i}$ are the nodal degrees of freedom obtained using either the proposed SGCSFEM method or by conventional FEM, and $U_{i}^{M C S}$ are the displacements obtained using Monte Carlo simulations.

\subsection{Stochastic two dimensional cantilever beam}

Consider a cantilever beam of length $L$ and width $D$ as shown in Figure 4. In this section, the stochastic static response and the free vibration is studied. In both the cases, the Young's modulus is assumed to be random. The Poisson's ratio is assumed to be deterministic with $\nu=0.3$ and a state of plane stress condition is assumed.

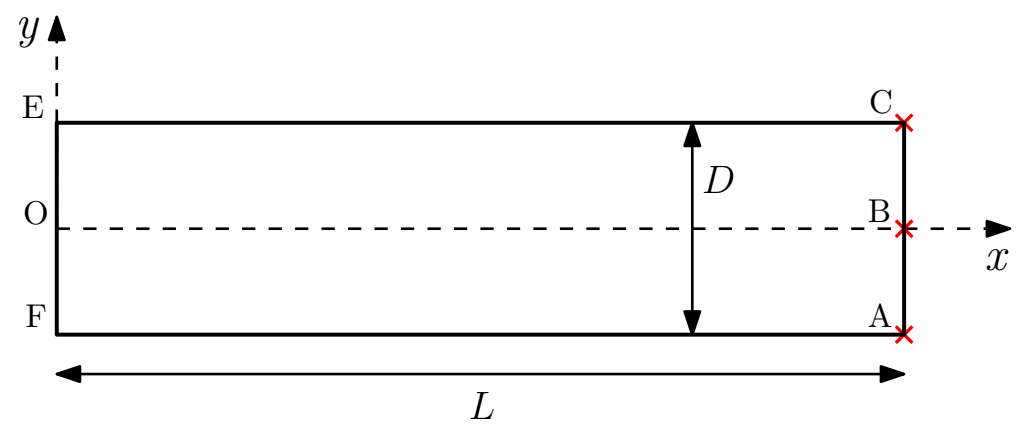

Fig. 4. Cantilever beam geometry

Static bending case with random $\mathbf{E}$ In this case, the statistical moments of maximum static deflection of a two dimensional cantilever beam under a deterministic parabolic shear loading is investigated. The geometry is length $L=8 \mathrm{~m}$, height $D=4 \mathrm{~m}$. The material properties are: mean value of Young's Modulus, $\langle E\rangle=20000$ 
$\mathrm{N} / \mathrm{m}^{2}$ with a coefficient of variation $\mathrm{COV}\left(\mathrm{COV}=\sigma_{E} / \mu_{E}\right)$ of 0.2 and the parabolic shear force $P=250 \mathrm{~N}$. The exact solutions for displacements are given by:

$$
\begin{aligned}
& u(x, y)=\frac{P y}{6 \bar{E} I}\left[(9 L-3 x) x+(2+\bar{\nu})\left(y^{2}-\frac{D^{2}}{4}\right)\right] \\
& v(x, y)=\frac{-P}{6 \bar{E} I}\left[3 \bar{\nu} y^{2}(L-x)+(4+5 \bar{\nu}) \frac{D^{2} x}{4}+(3 L-x) x^{2}\right]
\end{aligned}
$$

where $\mathrm{I}=D^{3} / 12$ is the moment of inertia, $\bar{E}=\frac{E}{1-\nu^{2}}, \bar{\nu}=\frac{\nu}{1-\nu}$. The left end of the beam E-O-F is subjected to analytical displacements given by Equation (5.2), whilst the right end of the beam A-B-C is subjected to a parabolic shear load with magnitude of $P=250 \mathrm{~N}$. Four levels of progressive mesh refinement is considered ( $8 \times 4,16 \times 8,32 \times 16,64 \times 32$ ). Table 2 compares the $L^{2}$ norm of the displacement at

Table 2. $L^{2}$ norm of the displacement at node $A$ of the cantilever beam: comparison of the CS-FEM with 4 sub-cells and FEM results with analytical solution

\begin{tabular}{ccccc}
\hline \multirow{2}{*}{ Mesh } & \multicolumn{2}{c}{ CS-FEM } & \multicolumn{2}{c}{ FEM } \\
\cline { 2 - 5 } & 4 SC & \% error & Q4 & \% error \\
\hline $8 \times 4$ & 0.488 & 0.94 & 0.447 & 9.25 \\
$16 \times 8$ & 0.491 & 0.26 & 0.480 & 2.46 \\
$32 \times 16$ & 0.492 & 0.07 & 0.489 & 0.64 \\
$64 \times 32$ & 0.492 & 0.02 & 0.491 & 0.17 \\
\hline
\end{tabular}

node $A$ (see Figure 4), obtained using a deterministic CS-FEM and the conventional FEM. From the tabulated results, it is clear that the solution provided by the CSFEM is within $1 \%$ from that provided by the analytical even with a coarse mesh of $8 \times 4$, while that given by the FEM is off by $\approx 10 \%$. Henceforth, for subsequent analysis, a mesh size of $8 \times 4$ will be used for the solution domain of the stochastic cantilever beam, unless otherwise specified.

Next, we compare the stochastic displacement solution obtained using the SGCS-FEM and the conventional FEM with the MCS. The Young's modulus is assumed to be random with an exponential covariance function (c.f. Equation (2.6)), with a correlation length of $\ell_{c}=10 \mathrm{~L}$ for which a maximum of 4 terms in the KL expansion of random field was found to be sufficient enough to capture the randomness. Tables 3-4 compares the statistical moments of $U_{x}$ and $U_{y}$ displacements, respectively, at nodes $A, B$ and $C$ (see Figure 4) using SGCS-FEM and FEM for various orders of PCE. The accuracy of the first two statistical moments (the mean and the standard deviation) obtained using the proposed method is compared with that of MCS using 10,000 samples. For brevity reasons, the MCS results are not repeated under the FEM columns. Following observations can be made from both the tables:

- Mean of displacements at points $A$ and $C$ are symmetrical about zero, due to their respective positions about the mid-plane of the geometry. Moreover, 
the standard deviations of the displacements at these points are mirror images of each other, for the above same reason.

- Mean $U_{x}$ displacement at point B turns out to be zero, as it can be seen by substituting its mid-plane coordinates $(x=L, y=0)$ into equation Equation (5.2).

- The statistical moments produced by the proposed SGCS-FEM formulation converges faster towards that of MCS, for the same random field representation and PC order expansions.

Figures 5 depicts the convergence of the error with mesh refinement for the SGCSFEM and the conventional FEM. Following observations can be made from these two plots:

- From the mean displacement error convergence plot, one can observe that the slope of the error obtained with SGCS-FEM is consistently lower than that of the FEM across the mesh sizes considered. Compounded with the presence of higher order PC expansions, the rate of convergence for the mean displacements increases dramatically when using the proposed method, especially in the region of finer mesh size.

- From displacement error convergence plot based on the standard deviation, the SGCS-FEM yields higher convergence rate towards MCS results over the conventional FEM, for the same order of PC expansions. However, such an advantage offered by the proposed method appears to be indistinguishable from that of the FEM, in fine mesh regions.

Table 3. Statistical moments of $U_{x}$ displacements at nodes marked A,B and C using SGCS-FEM (4 SC) and conventional FEM (Q4). Accuracy of the results are compared with MCS using 10000 samples

\begin{tabular}{|c|c|c|c|c|c|c|c|}
\hline & \multicolumn{3}{|c|}{ SGCS-FEM } & \multicolumn{3}{|c|}{ FEM } \\
\hline & & $\mathrm{A}$ & $\mathrm{B}$ & $\mathrm{C}$ & $\mathrm{A}$ & $\mathrm{B}$ & $\mathrm{C}$ \\
\hline \multirow{3}{*}{ Mean } & 1st order & -0.1548 & 0.0 & 0.1548 & -0.1413 & 0.0 & 0.1413 \\
\hline & 2nd order & -0.1554 & 0.0 & 0.1554 & -0.1418 & 0.0 & 0.1418 \\
\hline & MCS & -0.1572 & 0.0 & 0.1572 & & & \\
\hline \multirow[t]{3}{*}{ Std dev } & 1st order & $2.98 \times 10^{-2}$ & $1.77 \times 10^{-3}$ & $2.98 \times 10^{-2}$ & $2.72 \times 10^{-2}$ & $1.63 \times 10^{-3}$ & $2.71 \times 10^{-2}$ \\
\hline & 2nd order & $3.35 \times 10^{-2}$ & $2.06 \times 10^{-3}$ & $3.34 \times 10^{-2}$ & $3.07 \times 10^{-2}$ & $1.91 \times 10^{-3}$ & $3.06 \times 10^{-2}$ \\
\hline & MCS & $3.70 \times 10^{-2}$ & $2.12 \times 10^{-3}$ & $3.70 \times 10^{-2}$ & & & \\
\hline
\end{tabular}

Until now, the coefficient of variance was kept fixed at 0.2 . Next, the ability of the proposed method to capture the coefficient of variance in stochastic Quantity of Interest (QoI) as a function of $\mathrm{CoV}$ of an input random parameter will be studied. The CoV of random Young's Modulus is taken over the range from $5 \%$ $20 \%$. Figure 6 shows the mean along with the error bounds (defined by twice the standard deviation) for the $U_{y}$ displacement at node $A$ using a mesh of $8 \times 4$, as a 


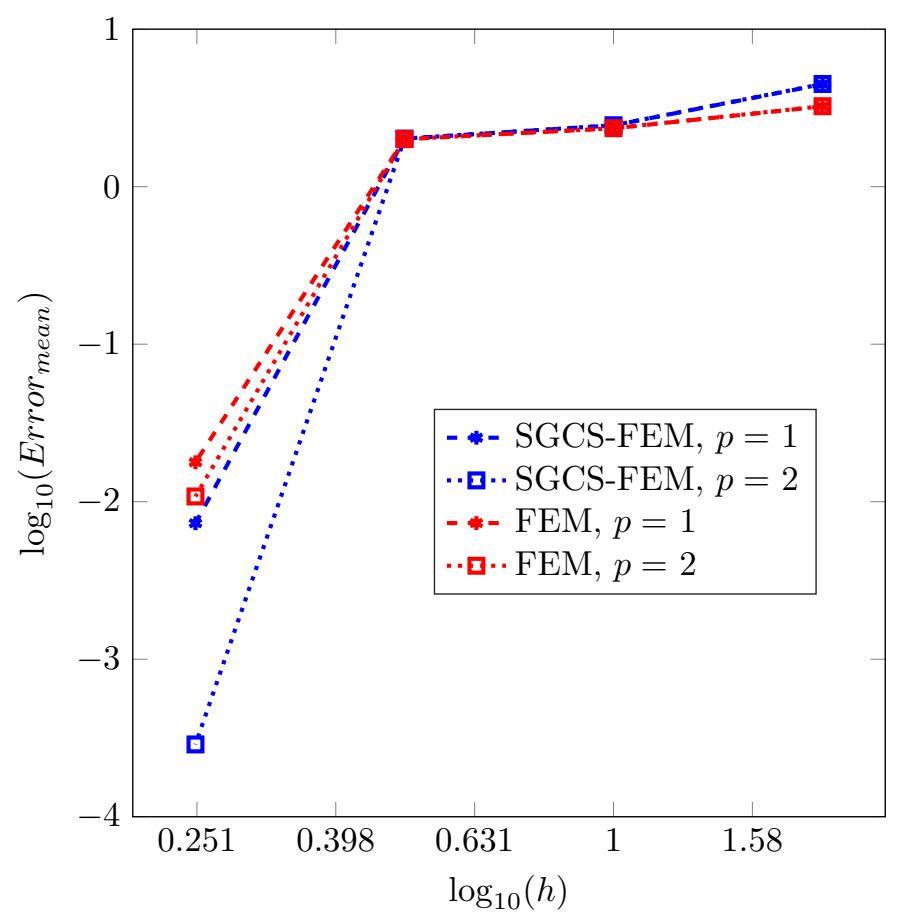

(a)

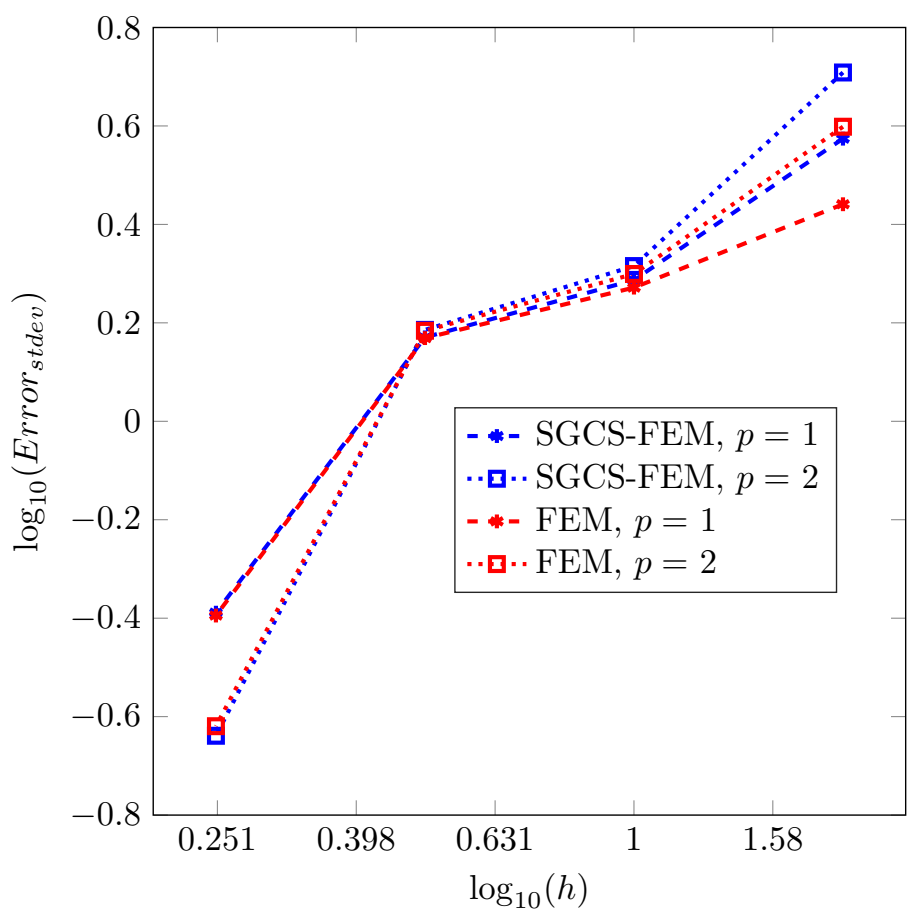

(b)

Fig. 5. Comparison of convergence rates between SGCS-FEM and FEM using: (a) the mean displacement norm definition and (b) the displacement norm definition based on standard deviation. 
Table 4. Statistical moments of $U_{y}$ displacements at nodes marked A,B and C using SGCS-FEM and conventional FEM. The accuracy of the results are compared with MCS using 10,000 samples

\begin{tabular}{|c|c|c|c|c|c|c|c|}
\hline & \multicolumn{3}{|c|}{ SGCS-FEM } & \multicolumn{3}{|c|}{ FEM } \\
\hline & & $\mathrm{A}$ & $\mathrm{B}$ & $\mathrm{C}$ & $\mathrm{A}$ & $\mathrm{B}$ & $\mathrm{C}$ \\
\hline \multirow[t]{3}{*}{ Mean } & 1st order & -0.4832 & -0.4839 & -0.4832 & -0.4428 & -0.4456 & -0.4428 \\
\hline & 2nd order & -0.4848 & -0.4856 & -0.4848 & -0.4443 & -0.4472 & -0.4443 \\
\hline & MCS & -0.4912 & -0.4913 & -0.4912 & & & \\
\hline \multirow[t]{3}{*}{ Std dev } & 1st order & $9.56 \times 10^{-2}$ & $9.57 \times 10^{-2}$ & $9.56 \times 10^{-2}$ & $8.85 \times 10^{-2}$ & $8.90 \times 10^{-2}$ & $8.85 \times 10^{-2}$ \\
\hline & 2nd order & $1.09 \times 10^{-1}$ & $1.09 \times 10^{-1}$ & $1.09 \times 10^{-1}$ & $1.01 \times 10^{-1}$ & $1.01 \times 10^{-1}$ & $1.01 \times 10^{-1}$ \\
\hline & MCS & $1.15 \times 10^{-1}$ & $1.15 \times 10^{-1}$ & $1.15 \times 10^{-1}$ & & & \\
\hline
\end{tabular}

function of $\mathrm{CoV}$ of the random Young's Modulus. From Figure 6, it can be inferred

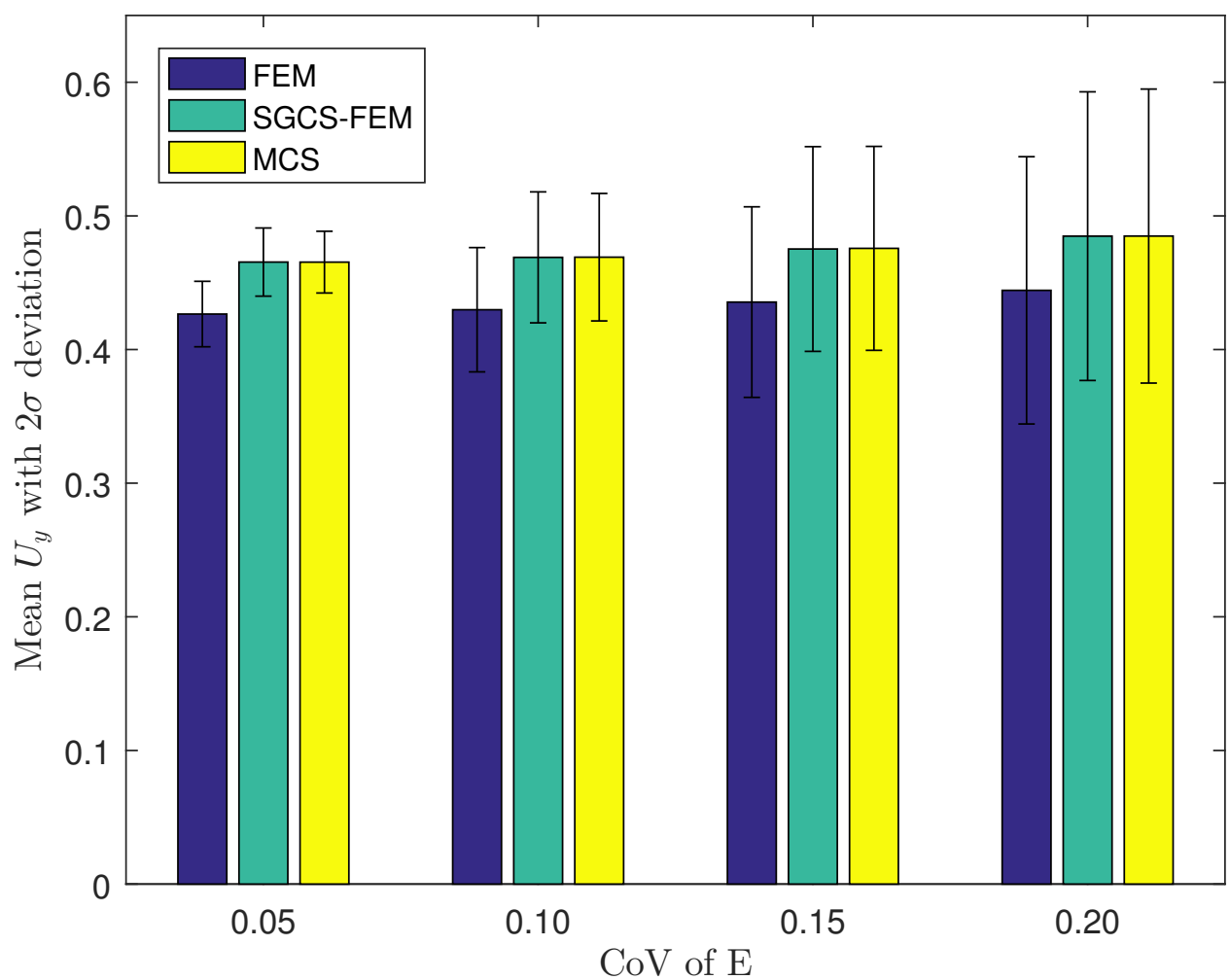

Fig. 6. Mean and variance of $U_{y}$ displacements at point A against the $\mathrm{CoV}$ of random Youngs' modulus

that the proposed SGCS-FEM reproduces similar results as that of MCS, both in 
mean and standard deviation of output displacements, over the considered range of input CoV. However, the FEM tends to consistently generate an under-predicted results, both in terms of mean and standard deviation, for the same mesh in solution domain.

Free vibration with random $\mathbf{E}$ Next, the stochastic vibration of a cantilever beam with $L=100 \mathrm{~mm}$ and $D=10 \mathrm{~mm}$ is studied. A plane stress condition is assumed considering a unit thickness into the plane. The Young's modulus is assumed to be random with mean value of $\langle E\rangle=2.1 \times 10^{4} \mathrm{Kgf} / \mathrm{mm}^{4}$ and coefficient of variation, $\mathrm{CoV}=0.2$. Poisson's ratio $\nu=0.3$ and mass density $\rho=8 \times 10^{-10}$ $\mathrm{Kgf} \mathrm{s}^{2} / \mathrm{mm}^{4}$. Using the Euler-Bernouilli beam theory, the first deterministic natural frequency of the beam is found to be $f_{1}=0.08276 \times 10^{4} \mathrm{~Hz}$. The domain is meshed with Q4 elements, with each element further subdivided into 4 subcells in case of SGCS-FEM. While the stiffness matrix is assembled using the smoothing technique, the lumped mass matrix, as in FEM, is adopted for stochastic vibration studies.

From a mesh convergence study, a mesh size of 50x5 was found to be sufficient enough, using CS-FEM, to reproduce the first natural frequency within $1 \%$ deviation from that of analytical values (see table 5).

\begin{tabular}{|c|c|c|c|c|}
\hline Number of elements & Number of nodes & SFEM (4 SC) & FEM (Q4) & Reference $^{29}$ \\
\hline \multirow[t]{4}{*}{$10 \times 1$} & 22 & 0.087 & 0.100 & \\
\hline & & 0.533 & 0.608 & \\
\hline & & 1.286 & 1.286 & \\
\hline & & 1.459 & 1.642 & \\
\hline \multirow[t]{4}{*}{$20 \times 2$} & 63 & 0.084 & 0.087 & 0.0926 \\
\hline & & 0.506 & 0.526 & 0.5484 \\
\hline & & 1.284 & 1.284 & 1.2832 \\
\hline & & 1.350 & 1.401 & 1.4201 \\
\hline \multirow[t]{4}{*}{$50 \times 5$} & 306 & 0.082 & 0.083 & 0.0844 \\
\hline & & 0.495 & 0.499 & 0.5051 \\
\hline & & 1.283 & 1.283 & 1.2828 \\
\hline & & 1.308 & 1.317 & 1.3258 \\
\hline
\end{tabular}

For an assumed correlation length of $\ell_{c}=12 \mathrm{~L}$ for the random field, five terms in the KL expansion was found to be sufficient enough to capture the decay in covariance function. The maximum order of $\mathrm{PC}$ expansions was chosen to be 1 . Table 6 summarizes the mean and the standard deviation of the first natural frequency obtained using the proposed SGCS-FEM and conventional FEM, using the stochastic inverse subspace method as detailed in algorithm 1. Meanwhile, Figure 7 
plots and compares the probability density function of the first natural frequency of the cantilever beam obtained using FEM and SGCS-FEM against that of MCS.

Table 6. Mean $\left(\times 10^{4} \mathrm{~Hz}\right)$ and standard deviation of the 1st natural frequency computed using different numerical techniques

\begin{tabular}{llcc}
\hline Numerical technique & & FEM & SGCS-FEM \\
\hline Mean $[\mathrm{Hz}]$ & 1st order PCE & 0.0827 & 0.0832 \\
& MCS & 0.0820 & \\
\multirow{2}{*}{ Std Dev $[\mathrm{Hz}]$} & 1st order PCE & $8.27 \times 10^{-3}$ & $8.31 \times 10^{-3}$ \\
& MCS & $8.39 \times 10^{-3}$ & \\
\hline
\end{tabular}

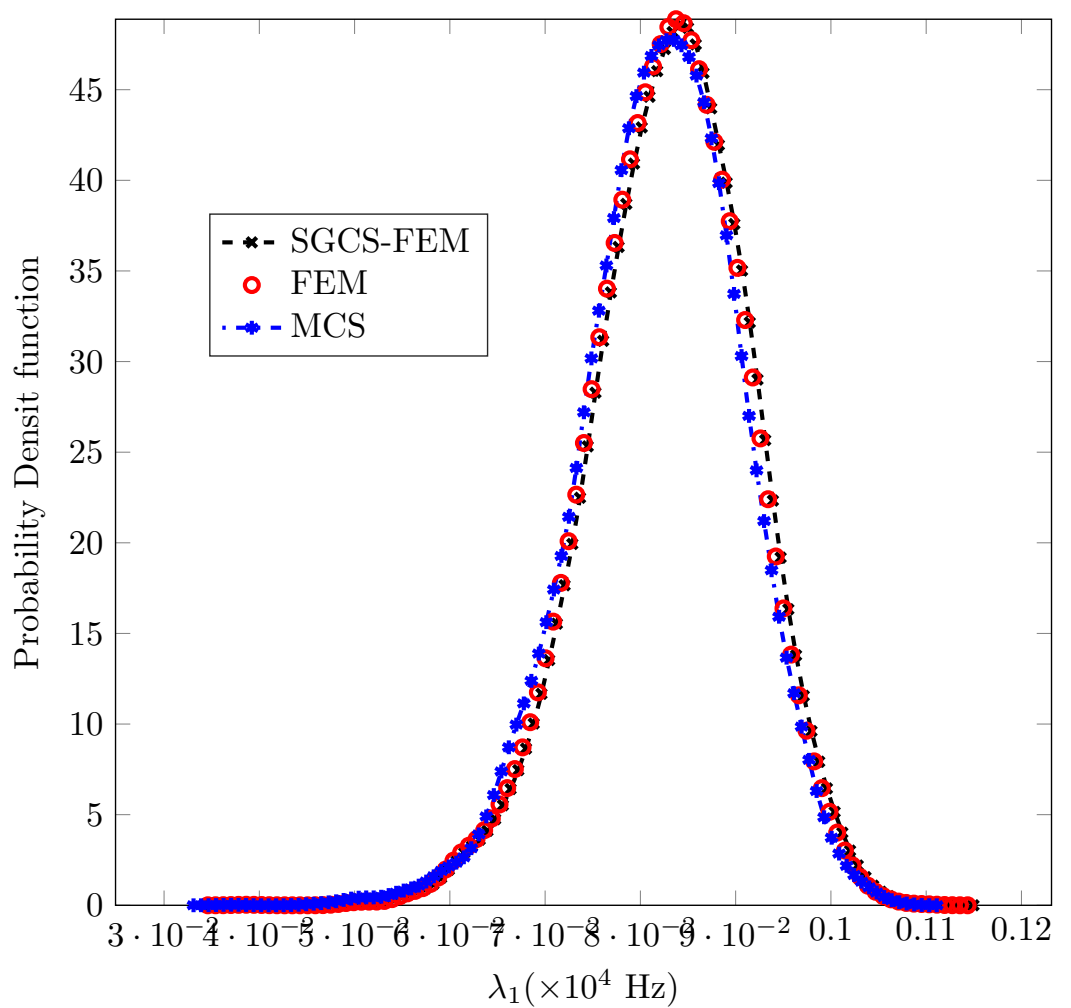

Fig. 7. Comparison of probability density function between MCS and KL-PCE using FEM and SGCS-FEM. Mesh size of $50 \times 5$, KLterms $=5, \ell_{c}=12 \mathrm{~L}$

From both the table 6 and the Figure 7, it is observed that the proposed SGCSFEM predicts almost the same statistical moments for the first natural frequency as that of MCS, producing an almost equivalent probability density function plot of the same. There exists not much of difference between the results produced by 
FEM and that of SGCS-FEM, as the converged mesh of $50 \times 5$ was used in the studies (refer back to table 5).

\subsection{Thick cylinder under internal pressure}

Consider a two dimensional hollow cylinder with an internal radius $r_{i}=1 \mathrm{~m}$ and an external radius $r_{o}=5 \mathrm{~m}$ subjected to an internal pressure $P_{i}=3 \times 10^{4} \mathrm{~Pa}$ on its inner surface $r_{i}$, while the outer surface $r_{o}$ is traction free (see Figure 8). Due to symmetry, only one-quarter of the domain is modeled and a structured mesh of 100 quadrilateral elements is chosen for this analysis (see Figure 8(b)). The Youngs' modulus is assumed to be random over the domain with a mean value of $\langle E\rangle=$ $3 \times 10^{7} \mathrm{~Pa}$ and $\mathrm{CoV}$ of 0.2 , with a correlation length equal to $r_{o}$. A plane stress condition is assumed for this problem.

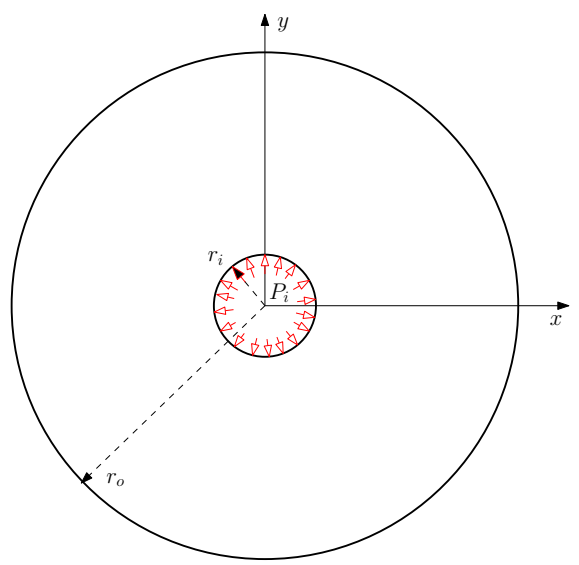

(a)

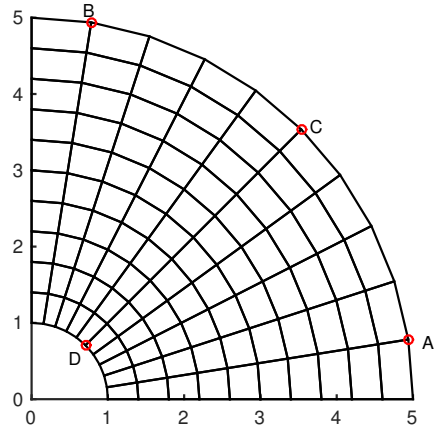

(b)

Fig. 8. Hollow cylinder under internal pressure: (a) Geometry and boundary conditions and (b) quarter model and a representative FE mesh.

Table 7. Displacement and standard deviation at selected target nodes along $x$ direction for $\mathrm{CoV}$ of 0.2

\begin{tabular}{llllll}
\hline Target points & & Node A & Node B & Node C & Node D \\
\hline Mean & First order of PCE & 0.4203 & 0.0668 & 0.3006 & 0.9573 \\
& Second order of PCE & 0.4211 & 0.0669 & 0.3011 & 0.9593 \\
& MCS & 0.4251 & 0.0673 & 0.3043 & 0.9664 \\
& & & & \\
Std Dev & First order of PCE & 0.0709 & 0.0133 & 0.0522 & 0.1640 \\
& Second order of PCE & 0.0776 & 0.0147 & 0.0569 & 0.1804 \\
& MCS & 0.0993 & 0.0157 & 0.0711 & 0.2259 \\
\hline
\end{tabular}

The number of KLterms chosen to represent the random field is fixed at 4, while the Hermite polynomial order is varied from 1 to 2 . A two dimensional exponential 
Table 8. Displacement and standard deviation at selected target nodes along $y$ direction for $\mathrm{CoV}$ of 0.2

\begin{tabular}{llllll}
\hline Target points & & Node A & Node B & Node C & Node D \\
\hline Mean & First order of PCE & 0.0668 & 0.4203 & 0.3006 & 0.9573 \\
& Second order of PCE & 0.0669 & 0.4211 & 0.3011 & 0.9593 \\
& MCS & 0.0673 & 0.4251 & 0.3043 & 0.9664 \\
& & & & \\
Std Dev & First order of PCE & 0.0133 & 0.0709 & 0.0522 & 0.1640 \\
& Second order of PCE & 0.0147 & 0.0776 & 0.0569 & 0.1804 \\
& MCS & 0.0157 & 0.0993 & 0.0711 & 0.2259 \\
\hline
\end{tabular}

covariance function is chosen to represent the randomness across the domain. Tables 7 - 8 compares the statistical response results at 4 different points $A, B, C$ and $D$, using the proposed SGCS-FEM formulation with the MCS using 10000 samples. It is clear from the table that the present formulation approximates the mean of the statistical response at all points to within $1 \%$ from that of the MCS. Moreover, the current formulation with a setting of KL terms as 4 and polynomial order as 2, was found to predict the standard deviation within $20 \%$ deviation from that predicted by the MCS. Furthermore, this relative difference is bound to decrease further with increasing polynomial order representation in random space.

\subsection{Three dimensional Cooks membrane}

As a last example, consider a three dimensional Cooks' membrane subject to end shear as shown in Figure 9. The applied end shear results in a deformation dominated by bending. The domain is discretized with eight noded hexahedral element and each element is further sub-divided into eight subcells. For this example, the Young's modulus is assumed to be random across the domain, with a mean value of $\langle E\rangle=20000$ and $\mathrm{CoV}=0.2$. Poisson ratio is taken to be $\nu=0.3$. The shear force loading, $\mathrm{F}$ is given a value of 500 and the correlation function is chosen to be of exponential in nature with the same correlation length of 10 times the maximum dimension for all the 3 mutually perpendicular directions.

The statistical moments in terms of the mean and the standard deviation of $U_{x}, U_{y}$ and $U_{z}$ at points marked $A$ and $B$ (see Figure 9) are first estimated by running the three dimensional model using 10000 random samples as part of the MCS. They are then compared with the ones computed using the proposed method. Tables 9- 11 tabulates the mean and the standard deviations of $U_{x}, U_{y}$ and $U_{z}$ displacements at points marked $A$ and $B$.

Comparing the results from the above table, one can see that the proposed method agrees well with that of deterministic MCS results within a maximum deviation of less than $2 \%$. 


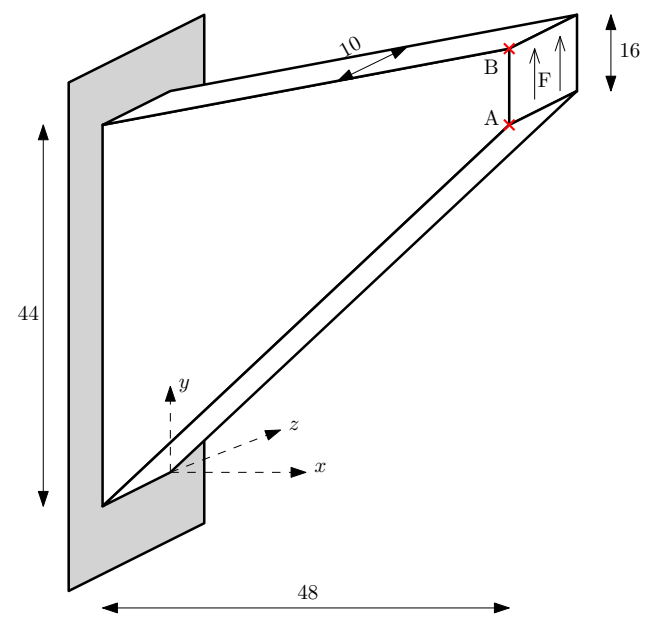

Fig. 9. Three dimensional Cooks membrane: geometry and boundary conditions

Table 9. Mean and standard deviation of $U_{x}$ displacements at selected target nodes

\begin{tabular}{llcc}
\hline Target points & & Node A & Node B \\
\hline Mean & First order of PCE & -0.1108 & -0.4363 \\
& Second order of PCE & -0.1111 & -0.4378 \\
& MCS & -0.1093 & -0.4347 \\
Std Dev & First order of PCE & $2.17 \times 10^{-2}$ & $8.50 \times 10^{-2}$ \\
& Second order of PCE & $2.45 \times 10^{-2}$ & $9.60 \times 10^{-2}$ \\
& MCS & $2.60 \times 10^{-2}$ & $10.4 \times 10^{-2}$ \\
\hline
\end{tabular}

Table 10. Mean and standard deviation of $U_{y}$ displacements at selected target nodes

\begin{tabular}{llcc}
\hline Target points & & Node A & Node B \\
\hline Mean & First order of PCE & 0.5626 & 0.5971 \\
& Second order of PCE & 0.5645 & 0.5991 \\
& MCS & 0.5606 & 0.5952 \\
Std Dev & First order of PCE & $1.10 \times 10^{-1}$ & $1.16 \times 10^{-1}$ \\
& Second order of PCE & $1.24 \times 10^{-1}$ & $1.31 \times 10^{-1}$ \\
& MCS & $1.34 \times 10^{-1}$ & $1.42 \times 10^{-1}$ \\
\hline
\end{tabular}

\section{Conclusions}

In this paper, the cell-based smoothed finite element technique is extended for solving stochastic PDEs with random field domains for the first time as a more efficient alternative to the conventional stochastic FEM. The performance of the proposed SGCS-FEM method in terms of the accuracy, was studied comprehensively using a few benchmark examples under static and free vibration modes. The accuracy of the method was correlated using the statistical moments obtained from the MCS. It 
Table 11. Mean and standard deviation of $U_{z}$ displacements at selected target nodes

\begin{tabular}{llcc}
\hline Target points & & Node A & Node B \\
\hline Mean & First order of PCE & -0.0477 & -0.0427 \\
& Second order of PCE & -0.0287 & -0.0237 \\
& MCS & -0.0055 & -0.0005 \\
Std Dev & First order of PCE & $1.90 \times 10^{-2}$ & $1.80 \times 10^{-2}$ \\
& Second order of PCE & $9.45 \times 10^{-3}$ & $8.82 \times 10^{-3}$ \\
& MCS & $1.32 \times 10^{-3}$ & $1.27 \times 10^{-4}$ \\
\hline
\end{tabular}

could be inferred that the proposed formulation yields commensurate results with that of the MCS. The influence of the input coefficient of variance on the statistical moments of the output response produced by the proposed method was studied in detail and compared with that of the MCS. It was observed that with a relatively coarser mesh, SGCS-FEM was able to reproduce the same mean and the standard deviation as that of the MCS for a range of input COV, while clearly outperforming the FEM consistently.

\section{Appendix A}

Analytical solutions of Equation (2.8) are readily available in the literature ${ }^{1}$ for simple triangular and exponential covariance function across a rectangular domain. However, for a random field with arbitrary covariance function across complex domains, the eigensolutions of Fredholm equation need to be computed numerically. In this work, the Galerkin method using finite element bases is adopted for the same. Within this framework, the $i^{\text {th }}$ eigenfunction $\theta_{i}$ is represented as a linear combination of finite element bases $\left\{\Phi_{k}\right\}_{k=1}^{N}$ with unknown nodal coefficients $d_{i k}$.

$$
h_{i}(\mathbf{x})=\sum_{k=1}^{N} d_{i k} \Phi_{k}(\mathbf{x})
$$

where $N$ stands for the number of nodes in the FE mesh. Substituting Equation (6.1) in Equation (2.8) and minimizing the error in the Galerkin sense, and upon further simplification, we get the following:

$\sum_{k=1}^{N} d_{i k}\left[\int_{\Omega} \int_{\Omega} \mathbf{R}_{h}\left(\mathbf{x}_{1}, \mathbf{x}_{2}\right) \Phi_{k}\left(\mathbf{x}_{\mathbf{2}}\right) \Phi_{j}\left(\mathbf{x}_{\mathbf{1}}\right) \mathrm{d} \Omega \mathrm{d} \Omega\right]-\gamma_{i} \sum_{k=1}^{N} d_{i k}\left[\int_{\Omega} \Phi_{k}\left(\mathbf{x}_{\mathbf{1}}\right) \Phi_{j}\left(\mathbf{x}_{\mathbf{1}}\right) \mathrm{d} \Omega\right]=0$

where $j$ goes from 1 to $\mathrm{N}$. The above relation can be written in the form of a generalized matrix eigenvalue problem as follows:

$$
\mathrm{AD}=\Lambda \mathrm{BD}
$$


where

$$
\begin{aligned}
\mathbf{A}_{j k} & =\int_{\Omega} \int_{\Omega} \mathbf{R}_{h}\left(\mathbf{x}_{1}, \mathbf{x}_{2}\right) \Phi_{k}\left(\mathbf{x}_{\mathbf{2}}\right) \Phi_{j}\left(\mathbf{x}_{\mathbf{1}}\right) \mathrm{d} \Omega \mathrm{d} \Omega, \\
\mathbf{B}_{j k} & =\int_{\Omega} \Phi_{k}\left(\mathbf{x}_{\mathbf{1}}\right) \Phi_{j_{1}}(\mathbf{x}) \mathrm{d} \Omega \\
\mathbf{D}_{j i} & =d_{j i} \quad \boldsymbol{\Lambda}_{j i}=\delta_{j i} \gamma_{i}
\end{aligned}
$$

where $\mathbf{D}, \boldsymbol{\Lambda}$ are the eigenvectors and eigenvalues, respectively, of size $N \times N$. The integrals in matrices $\mathbf{A}$ and $\mathbf{B}$ are computed by Gauß quadrature rule. Since we are dealing with two dimensional structures, the integral in matrix $\mathbf{A}$ is a quadruple integration, whereas for $\mathbf{B}$, it is a double integral.

\section{References}

1. Roger G. Ghanem and Pol D. Spanos. Stochastic finite elements: a spectral approach. Springer-Verlag, New York, 1991.

2. M. Kleiber and T. D. Hien. The stochastic finite element method (basic perturbation technique and computer implementation). Applied Stochastic Models and Data Analysis, 10(4):297-297, 1992.

3. J. E. Hurtado and A. H. Barbat. Monte carlo techniques in computational stochastic mechanics. Archives of Computational Methods in Engineering, 5(1):3, 1998.

4. Fumio Yamazaki, Masanobu Shinozuka, and Gautam Dasgupta. Neumann expansion for stochastic finite element analysis. Journal of Engineering Mechanics, 114(8):1335$1354,1988$.

5. MarcinKaminski. Stochastic second-order perturbation approach to the stress-based finite element method. International Journal of Solids and Structures, 38(21):38313852, 2001.

6. Sachin K.Sachdeva, Prasanth B.Nair, and Andy J.Keane. Comparative study of projection schemes for stochastic finite element analysis. Computer Methods in Applied Mechanics and Engineering, 195(19):2371-2392, 2006.

7. Roger Ghanem and Pol D Spanos. Polynomial chaos in stochastic finite elements. Journal of Applied Mechanics, 57(1):197-202, 1990.

8. R. Ghanem and P.D. Spanos. A stochastic galerkin expansion for nonlinear random vibration analysis. Probabilistic Engineering Mechanics, 8(3):255-264, 1993.

9. R. Ghanem and W. Brzakala. Stochastic finite-element analysis of soil layers with random interface. Journal of Engineering Mechanics, 122(4):361-369, 1996.

10. Maciej Anders and Muneo Hori. Stochastic finite element method for elasto-plastic body. International Journal for Numerical Methods in Engineering, 46(11):1897-1916, 199.

11. Wolfgang Betz, Iason Papaioannou, and Daniel Straub. Numerical methods for the discretization of random fields by means of the karhunenloéve expansion. Computer Methods in Applied Mechanics and Engineering, 271:109-129, 2014.

12. X.Y.Long, C.Jiang, C.Yang, X.Han, W.Gao, and J.Liu. A stochastic scaled boundary finite element method. Computer Methods in Applied Mechanics and Engineering, 308:23-46, 2016.

13. C.O.Arun, B.N.Rao, and S.M.Srinivasan. Stochastic meshfree method for elastoplastic damage analysis. Computer Methods in Applied Mechanics and Engineering, 199(37):2590 - 2606, 2010. 
14. Keyan Li, Wei Gao, Di Wu, Chongmin Song, and Taicong Chen. Spectral stochastic isogeometric analysis of linear elasticity. Computer Methods in Applied Mechanics and Engineering, 332:157 - 190, 2018.

15. Jiun-Shyan Chen, Cheng-Tang Wu, Sangpil Yoon, and Yang You. A stabilized conforming nodal integration for galerkin mesh-free methods. International Journal for Numerical Methods in Engineering, 50(2):435-466, 2001.

16. G.R.Liu, W.Zeng, and H.Nguyen-Xuanb. Generalized stochastic cell-based smoothed finite element method (gs-cs-fem) for solid mechanics. Finite Elements in Analysis and Design, 63:51-61, 2013.

17. W Zeng and G. R. Liu. Smoothed finite element methods (s-fem): An overview and recent developments. Archives of Computational Methods in Engineering, 25(2):397$435,2018$.

18. X.B.Hua, X.Y.Cui, H.Feng, and G.Y.Li. Stochastic analysis using the generalized perturbation stable node-based smoothed finite element method. Engineering Analysis with Boundary Elements, 70:40-55, 2016.

19. A generalized probabilistic edge-based smoothed finite element method for elastostatic analysis of reissnermindlin plates. Applied Mathematical Modelling, 53:333 -352, 2018.

20. A stochastic perturbation edge-based smoothed finite element method for the analysis of uncertain structural-acoustics problems with random variables. Engineering Analysis with Boundary Elements, 80:116-126, 2017.

21. Bruno Sudret, Armen Der Kiureghian, Bruno Sudret, and Armen Der Kiureghian. Stochastic finite element methods and reliability: A state-of-the-art report. Technical report, 2000.

22. ChunChing Li and A. Der Kiureghian. Optimal discretization of random fields. Journal of Engineering Mechanics, 119(6):1136-1154, 1993.

23. Jeong Wahn Yoo, Brian Moran, and Jiun-Shyan Chen. Stabilized conforming nodal integration in the natural-element method. International Journal for Numerical Methods in Engineering, 60(5):861-890, 2004.

24. Hung Nguyen-Xuan, Stéphane Bordas, and Hung Nguyen-Dang. Smooth finite element methods: convergence, accuracy and properties. International Journal for $\mathrm{Nu}$ merical Methods in Engineering, 74(2):175-208, 2008.

25. Sundararajan Natarajan, Ean Tat Ooi, Irene Chiong, and Chongmin Song. Convergence and accuracy of displacement based finite element formulations over arbitrary polygons: Laplace interpolants, strain smoothing and scaled boundary polygon formulation. Finite Elements in Analysis and Design, 85:101-122, 2014.

26. M.FPellissetti and R.GGhanem. Iterative solution of systems of linear equations arising in the context of stochastic finite elements. Advances in Engineering Software, 31(8):607-616, 2000.

27. Roger G. Ghanem and Robert M. Kruger. Numerical solution of spectral stochastic finite element systems. Computer Methods in Applied Mechanics and Engineering, 129(3):289-303, 1996.

28. Howard C. Elman. Inverse subspace iteration for spectral stochastic finite element methods. 2015.

29. Toshio Nagashima. Node-by-node meshless approach and its applications to structural analyses. International Journal for Numerical Methods in Engineering, 46(3):341-385, 1999. 